\title{
Condições associadas a atividade de planejamento nos centros de especialidades odontológicas*
}

Conditions Associated with Activity Planning in Dental Specialty Centers

Condiciones asociadas a la actividad de planeación en centros de especialidades odontológicas

Fecha de recepción: 18-09-2018 | Fecha de aceptación: 19-03/2019

\author{
EdSON Hilan Gomes de LuCENA \\ Universidade Federal de Pernambuco, Recife, Pernambuco, Brasil. ehglucena@gmail.com; \\ https://orcid.org/0000-0003-3431-115X
}

\section{Carolina Dantas Rocha Xavier de Lucena}

Universidade Federal de Pernambuco, Recife, Pernambuco, Brasil. krolrocha@yahoo.com.br; https://orcid.org/0000-0002-4652-993

\section{Paulo Savio Angeiras de Goes}

Universidade Federal de Pernambuco, Recife, Pernambuco, Brasil. paulosaviogoes@gmail.com;

https://orcid.org/0000-0002-6708-0450 


\section{MARia Fátima de Sousa}

Universidade de Brasília, Brasília, DF, Brasil. fatimasousa@ unb.br; https://orcid.org/0000-0001$\underline{6949-9194}$

* Artigo de pesquisa original

Correspondencia: $\quad$ ehglucena@gmail.com; $\quad$ krolrocha@yahoo.com.br; paulosaviogoes@gmail.com; fatimasousa@unb.br

doi: https://doi.org/10.11144/Javeriana.uo38-80.caap

Cómo citar: de Lucena EHG, de Lucena CDR, de Goes PSA, de Sousa MF. Condições associadas a atividade de planejamento nos centros de especialidades odontológicas. Univ Odontol. 2019 enejun; 38(80). https://doi.org/10.11144/Javeriana.uo38-80.caap

\section{RESUMO}

Antecedentes: O planejamento nos serviços de saúde visa especificamente melhorar o estado de saúde de uma determinada população, garantindo o acesso com equidade e justiça, bem como agilizar a resposta do sistema de saúde às necessidades percebidas pela comunidade. Objetivo: Analisar a associação entre o relato da prática do planejamento nos Centros de Especialidades Odontológicas (CEO) com as características do município, do serviço e do gestor do serviço. Método: É um estudo de natureza quantitativa, onde se utilizou o banco de dados da Avaliação 
Externa do primeiro ciclo do Programa Nacional de Melhoria do Acesso e da Qualidade dos CEO (PMAQ-CEO), relativos ao módulo de entrevista com o Gerente e um Cirurgião-Dentista do CEO, além de verificação de documentos. A análise ocorreu do ponto de vista descritiva e analítica para a qual aplicou-se o teste qui-quadrado de Pearson, considerando o nível de significância de $5 \%$. Resultados: A maioria dos CEO que relataram ter realizado alguma atividade de planejamento, nos últimos 12 meses, está na Região Centro-Oeste $(88,7 \%)$ e Sul (82,1 \%), em municípios com menor cobertura de saúde bucal da estratégia Saúde da Família e com alto Índice de Desenvolvimento Humano (IDHM). Porém não houve correlação com o porte populacional dos municípios. Conclusões: Os CEO que possuem gerente, com um tempo de atuação no serviço maior do que 2 anos e que tenha uma formação complementar na área de gestão pública ou de saúde coletiva tendem a realizar atividades de planejamento.

\section{Palavras-chave}

atenção secundária à saúde; gestão em saúde; planejamento em saúde; saúde bucal; serviços de saúde

\section{Áreas temáticas}

odontologia; odontologia social e preventiva; saúde pública

\section{ABSTRACT}

Background: Planning in health services specifically aims to improve the health status of a given population, ensuring access with fairness and justice, as well as streamlining the response of the 
health system to the needs perceived by the community. Purpose: Analyze the association between the practice of planning inside the Specialized Dental Centers (SDC) and the city, the service and the service manager characteristics. Methods: It is a quantitative study, in which was used database of the first cycle of the External Evaluation of the National Program for Access and Quality Improvement of SDC (NPAQI-SDC) as well as a documental verification. The analysis was both descriptive and analytical to which was applied Pearson's chi-square test, considering $5 \%$ the level of significance. Results: That most of the SDC that reports to have done some planning activity in the past 12 months, are in the Midwest (88.7\%) and South areas (82.1\%), in municipalities with lower oral health coverage of the Family Health Strategy and with a high Municipal Human Development Index (MHDI). Conclusion: Although there was no correlation with the population size of the municipalities, the SDC whose manager, had at least two years of education in public management area or public health tend to perform planning activities.

\section{Keywords}

health management; health planning; health services; oral health; secondary care

\section{Thematic fields}

dentistry; public health; social and preventive dentistry

\section{RESUMEN}

Antecedentes: La planificación en los servicios de salud busca específicamente mejorar el estado de salud de una determinada población, garantizando el acceso con equidad y justicia, así como 
agilizar la respuesta del sistema de salud a las necesidades percibidas por la comunidad. Objetivo: Analizar la asociación entre el relato de la práctica de la planificación en los Centros de Especialidades Odontológicas (CEO) con las características del municipio, del servicio y del gestor del servicio. Método: Es un estudio de naturaleza cuantitativa, donde se utilizó el banco de datos de la Evaluación externa del primer ciclo del Programa Nacional de Mejora del Acceso y de la Calidad de los CEO (PMAQ-CEO), relativos al módulo de entrevista con el Gerente y un Gerente Cirujano-Dentista del CEO, además de verificación de documentos. El análisis ocurrió desde el punto de vista descriptivo y analítico para la cual se aplicó la prueba qui-cuadrada de Pearson, considerando el nivel de significancia del 5\%. Resultados: La mayoría de los CEO que reportaron haber realizado alguna actividad de planificación, en los últimos 12 meses, está en la Región Centro-Oeste $(88,7 \%)$ y Sur $(82,1 \%)$, en municipios con menor cobertura de salud bucodental

estrategia de Salud de la Familia y con alto Índice de Desarrollo Humano (IDHM). Pero no hubo correlación con el porte poblacional de los municipios. Conclusiones: Los CEO que poseen gerente, con un tiempo de actuación en el servicio mayor de 2 años y que tenga una formación complementaria en el área de gestión pública o de salud colectiva tienden a realizar actividades de planificación.

\section{Palabras clave}

atención secundaria a la salud; gestión de la salud; planeación en salud; salud bucal; servicios de salud

\section{Áreas temáticas}

odontología; odontología social y preventiva; salud pública 


\section{INTRODUÇÃO}

Para Noronha, Lima e Machado (2004) (1), o planejamento está entre as quatro grandes macrofunções da gestão. A preocupação com a discussão do planejamento e iniciativas de institucionalizá-lo como componente imprescindível da gestão sempre esteve presente, desde a concepção do Sistema Único de Saúde (SUS). Entretanto, existem particularidades que se colocam de forma emergente, mesmo com os avanços, e que constituem desafios para a efetivação do planejamento em saúde, enquanto instrumento da gestão no SUS (2).

O planejamento pode ser entendido como “...onde se que ir, como chegar lá e a programação...” (3). É uma ferramenta de trabalho da gestão utilizada para tomar decisões, direcionar a prestação de serviços de saúde e organizar as ações de forma lógica e racional, de modo a garantir os melhores resultados e a realização dos objetivos de uma sociedade, com os menores custos e no menor prazo possível $(4,5)$. Portanto, compreende a definição de objetivos futuros e as respectivas linhas de ação para atingi-los (6). Conforme Matus (1993) (7), planejamento é “o cálculo que precede e preside a ação", em um processo permanente de reflexão e análise para escolha de alternativas que permitam alcançar determinados resultados desejados no futuro. Um dos pontos fortes do planejamento está em sua aplicação como um agente de mudança (8). Portanto o denominador comum das diversas abordagens para definir "planejamento" é a sua "preocupação com a tomada de decisões relativas ao futuro" (9). 
O planejamento nos serviços de saúde visa especificamente melhorar o estado de saúde de uma determinada população, garantindo o acesso com equidade e justiça, bem como agilizar a resposta do sistema de saúde às necessidades percebidas pela comunidade. $\mathrm{O}$ alcance deste objetivo se dará através da prestação de serviços de saúde eficientes e eficazes, tendo em conta os recursos disponíveis e os meios e métodos de cuidados de saúde disponíveis (10).

A prestação de serviços de saúde ocorre em um ambiente cada vez mais dinâmico que sofre influências por meio da expectativa da comunidade, das prioridades do governo e os avanços tecnológicos (3). Nesse contexto o planejamento deve ser um 'instrumento vivo' que realmente acompanhe o dia a dia da produção de saúde, a fim de que as exigências impostas pela sociedade contemporânea, identificadas comumente como "incêndios", não se sobreponham às ações e aos compromissos assumidos coletivamente (11).

Ao considerar o planejamento como um dispositivo é concordar que este é um elemento disparador de uma série de acontecimentos/eventos que findam por convergir nos objetivos esperados, sejam eles uma transformação ou manutenção da ordem. É com este respaldo que o planejamento se estabelece, no campo da Saúde Coletiva, na perspectiva de assegurar o acesso dos cidadãos e oferecer serviços de alta qualidade a comunidade $(12,13)$.

Segundo Vieira (2009) (2), a ausência do planejamento, faz com que as atividades sejam executadas por inércia. Os serviços de saúde funcionam de forma desarticulada, sendo apenas orientados pela noção de qual seja seu papel e esta noção é produzida de acordo com a visão de mundo de cada gestor e colaborador ou de seu entendimento sobre as diretrizes estabelecidas pela 
política setorial do Estado. Consequentemente subjetiva-se, fragmenta-se e desordena-se o funcionamento do sistema de saúde na medida de tantas quantas visões e, consequentemente, modos de operação existam. Uma vez que a ausência de um entendimento objetivo e comum de onde se pretende chegar, cada profissional conduzirá e realizará suas atividades a sua maneira. Com tantos caminhamos em diversos sentidos, os avanços de uns se neutralizam pelos retrocessos de outros. Sendo assim não haverá ganho de qualidade e certamente os recursos serão empregados de forma menos eficiente.

Com objetivo de induzir os gestores e os profissionais dos Centros de Especialidades Odontológicas (CEO) a ampliar o acesso e a melhorar a qualidade dos serviços especializados de saúde bucal oferecidos aos cidadãos do território, o Ministério da Saúde implantou o Programa Nacional de Melhoria do Acesso e da Qualidade dos Centros de Especialidades Odontológicas (PMAQ-CEO). Para isso, propões um conjunto de estratégias de qualificação, acompanhamento e avaliação do trabalho dos CEO (14). Segundo Pisco (2006) (15), a finalidade de políticas de avaliação e de melhoria de qualidade não pode ser apenas para demonstrar os problemas, nem propor soluções para os problemas detectados, mas produzir mudanças apropriadas que conduzam à melhoria da qualidade dos cuidados prestados. O objetivo não é outro senão a identificação, a avaliação e a correção de situações potencialmente melhoráveis.

Os CEO são estabelecimentos públicos de saúde, classificados como Clínica Especializada/Ambulatório de Especialidade habilitados pelo Ministério da Saúde. Devem ofertar à população serviços de diagnóstico bucal, com ênfase em detectar câncer de boca, serviços de periodontia especializada, de cirurgia oral menor dos tecidos moles e duros e nos de endodontia e 
atendimento a pacientes com necessidades especiais (16). Os tratamentos oferecidos nos CEO são uma continuidade do cuidado prestado pela atenção básica a qual é responsável pelo primeiro atendimento e ordenadora da Rede. É importante destacar que a organização de uma rede hierarquizada de serviços é um dos recursos do planejamento para propiciar o acesso da população aos serviços de saúde com universalidade, equidade e integralidade (17).

A escolha desse eixo de investigação se justifica pela compreensão de que o planejamento é uma ferramenta estratégica de gestão para melhoria da qualidade da atenção à saúde e por sua contribuição ao subsídio da reflexão da organização de processos de trabalho complexos $(18,19)$. Nesse contexto, o presente estudo objetivou analisar a associação entre o relato da prática do planejamento, nos últimos 12 meses, nos Centros de Especialidades Odontológicas com as características do município, do serviço e do gestor do serviço.

\section{MATERIAIS E MÉTODOS}

O presente estudo é de natureza quantitativa, descritiva e analítica, o qual utilizou o banco de dados do primeiro ciclo do PMAQ-CEO, referente ao Módulo II - Entrevista com Gerente do CEO, com Cirurgião-Dentista do CEO e Verificação de Documentos, cuja coleta foi realizada no primeiro semestre de 2014 .

Foram analisados os dados de todos os Centros de Especialidades Odontológicas (CEO) coletados in loco durante a etapa de Avaliação Externa do Programa. Foram excluídos da amostra do 
Programa os CEO que estavam fechados, em reforma, que foram desabilitados pelo Ministério da Saúde ou que se recusaram a participar da avaliação.

Para compreender o planejamento realizado pelos gerentes e profissionais dos Centros de Especialidades Odontológicas (CEO), as características do serviço e do gestor do serviço foram selecionadas as seguintes questões, respectivamente: se foi realizado, nos últimos 12 meses, alguma atividade de planejamento das ações do CEO; se é realizado o monitoramento e análises das metas estabelecidas para cada especialidade ofertada no CEO; se foi realizado algum processo de autoavaliação pela Equipe do CEO nos últimos seis meses; se há gerente no serviço; o tempo de atuação do gerente no CEO; e se o mesmo tem ou está em formação complementar. Todas estas questões são específicas das subdimensões: 'Informações sobre o entrevistado (Gerente do CEO)', 'Formação e qualificação dos profissionais do CEO' e 'Planejamento e ações da gestão para organização do processo de trabalho do CEO’.

Estas foram associadas às variáveis contextuais do município representadas pelos indicadores socioeconômicos e de cobertura, a saber: Região do País; Porte Populacional, referente a 2013, obtido do Instituto Brasileiro de Geografia e Estatística (IBGE); Estimativa de cobertura populacional de Equipe de Saúde Bucal da Estratégia Saúde da Família, referente a 2013, disponível no Histórico de Cobertura da Saúde da Família publicado pelo Departamento de Atenção Básica do Ministério da Saúde; e o Índice de Desenvolvimento Humano Municipal (IDHM), Censo Demográfico de 2010, divulgado pelo Programa das Nações Unidas para o Desenvolvimento (PNUD). 
As variáveis foram agrupadas em três componentes de análises, da forma a seguir, e testadas a associação com a variável de realização de alguma atividade de planejamento, nos últimos 12 meses.

- Características Avaliativas do Município: Região, Porte Populacional, Proporção de cobertura populacional estimada de saúde bucal na Estratégia Saúde da Família (dicotomizada pela mediana: 63) e Índice de Desenvolvimento Humano Municipal (IDHM) (dicotomizado entre: $\geq$ $0,7=$ "alto e muito alto" e $<0,7=$ "médio, baixo e muito baixo");

- Características Avaliativas do Serviço: Tipo do CEO, Realização de algum processo de autoavaliação, nos últimos 6 meses, Realização do monitoramento e análises das metas das especialidades; e

- Características Avaliativas do Gestor do Serviço: Existência de gerente no CEO, Tempo de atuação do Gerente no CEO e Formação complementar do Gerente do CEO.

Dessa forma, no que se diz respeito às variáveis analisadas no estudo, tivemos como variável dependente a realização de alguma atividade de planejamento, nos últimos 12 meses. E como variáveis independentes aquelas relacionadas às características dos municípios, dos serviços e dos gestores dos serviços.

A associação entre as variáveis foi verificada com teste qui-quadrado de Pearson, considerando o nível de significância estatística de $5 \%$. Todos os testes estatísticos foram realizados através do 
software Statistic Package for the Social Sciences - SPSS versão 13.0 (SPSS Inc., Estados Unidos).

A avaliação externa do PMAQ-CEO foi conduzida dentro dos padrões exigidos pela Declaração de Helsinque e aprovada pelo Conselho de Ética em Pesquisa do Centro de Ciências da Saúde da Universidade Federal de Pernambuco sob o registro no 740.974 e CAAE 23458213.0.0000.5208.

\section{RESULTADOS}

No total, 930 CEO concluíram a etapa da Avaliação Externa do primeiro ciclo do PMAQ-CEO e $54(5 \%)$ foram excluídos considerando os critérios descritos anteriormente. Os quais estão distribuídos na Tabela 1 segundo as características avaliativas do Município, do Serviço e do Gestor do Serviço.

A maior concentração dos CEO foi na Região Nordeste $(38,2 \%)$, seguida das Regiões Sudeste (36,2\%), Sul (12,6\%), Centro-Oeste (6,7\%) e Norte (6,3\%). Quanto ao porte populacional, 39,4 \% localizam-se em municípios com mais de 100 mil habitantes. A maior parte dos CEO (59,2 \%) encontram-se em municípios que apresentam uma proporção de cobertura populacional estimada de saúde bucal na Estratégia Saúde da Família menor do que $63 \%$. E quando consideramos o Índice de Desenvolvimento Humano Municipal (IDHM), 59,5 \% dos serviços especializados em saúde bucal (CEO) estão em municípios com alto/muito alto IDHM (Tabela 1). 
Considerando a classificação dos CEO, 50,6 \% foram habilitados pelo Ministério da Saúde como tipo II, isto é, devem dispor em sua estrutura física de 4 a 6 cadeiras odontológicas, com uma oferta de carga horária clínica total de 160 a 360 horas/semanais. No que diz respeito a realização, nos últimos 12 meses, de alguma atividade de planejamento, 77,7\% dos entrevistados declaram durante a entrevista da avaliação externa que realizaram. E, quanto ao monitoramento e análise das metas de produção estabelecidas pelo Ministério da Saúde, por meio de portaria, para cada especialidade, apenas 14,6 \% afirmaram que não realizam (tabela 1).

Somente $12,3 \%$ dos serviços não apresentam gerente. Sendo que dos $87,7 \%$ que informam ter este profissional, apenas em $50 \%$ o mesmo atua exclusivamente na função gerente e não divide sua carga horária com atividades/atendimentos clínicos disponibilizando assim um maior tempo para atuar em fatores determinantes para atingir os objetivos organizacionais, mediante planejamento, coordenação, direção e controle. Considerando o tempo de atuação do gerente no CEO, 60,7\% atuam a menos de 2 anos no cargo, isto reflete o alto índice de rotatividade dos profissionais de saúde nos serviços públicos. Quanto a formação complementar desses profissionais, um pouco mais de um terço $(33,3 \%)$ tem formação em gestão pública $(14,7 \%)$ ou em saúde coletiva $(18,6 \%)$ (tabela 1). 
TABELA 1

DisTRIBUIÇÃO DOS CENTROS DE ESPECIALIDADES ODONTOLÓGICAS AVALIADOS NO PMAQ-CEO, SEGUNDO AS CARACTERÍSTICAS AVALIATIVAS, BRASIL

\begin{tabular}{|c|c|c|c|c|}
\hline \multicolumn{3}{|c|}{ Características Avaliativas } & \multirow{2}{*}{$\begin{array}{l}\mathbf{n} \\
59\end{array}$} & \multirow{2}{*}{$\frac{\%}{6,3}$} \\
\hline \multirow{17}{*}{ 总 } & \multirow{6}{*}{ 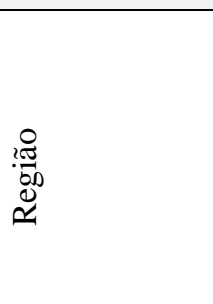 } & Norte & & \\
\hline & & Centro-Oeste & 62 & 6,7 \\
\hline & & Sul & 117 & 12,6 \\
\hline & & Sudeste & 337 & 36,2 \\
\hline & & Nordeste & 355 & 38,2 \\
\hline & & Total & 930 & 100 \\
\hline & \multirow{5}{*}{ ֻ. } & $0-30.000$ & 233 & 25,1 \\
\hline & & $30.001-50.000$ & 160 & 17,2 \\
\hline & & $50.001-100.000$ & 171 & 18,4 \\
\hline & & $>100.000$ & 366 & 39,4 \\
\hline & & Total & 930 & 100 \\
\hline & \multirow{3}{*}{ 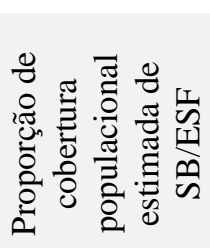 } & $\leq 63 \%$ & 551 & 59,2 \\
\hline & & $>63 \%$ & 379 & 40,8 \\
\hline & & Total & 930 & 100 \\
\hline & \multirow{3}{*}{$\underset{⿱ 乛}{\stackrel{1}{⿴ 囗 十}}$} & $<0,7$ & 377 & 40,5 \\
\hline & & $\geq 0,7$ & 553 & 59,5 \\
\hline & & Total & 930 & 100 \\
\hline \multirow{10}{*}{ 懖 } & \multirow{4}{*}{$\begin{array}{l}0 \\
0 \\
0 \\
0 \\
0 \\
\circ \\
\stackrel{1}{\ddots 1}\end{array}$} & 1 & 349 & 37,5 \\
\hline & & 2 & 470 & 50,6 \\
\hline & & 3 & 111 & 11,8 \\
\hline & & Total & 930 & 100 \\
\hline & \multirow{3}{*}{ 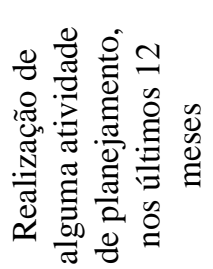 } & Não & 207 & 22,3 \\
\hline & & Sim & 723 & 77,7 \\
\hline & & Total & 930 & 100 \\
\hline & \multirow{3}{*}{ 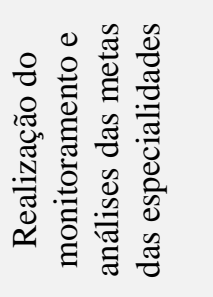 } & Não & 136 & 14,6 \\
\hline & & Sim & 794 & 85,4 \\
\hline & & Total & 930 & 100 \\
\hline \multirow{3}{*}{ 윌 } & \multirow{3}{*}{ 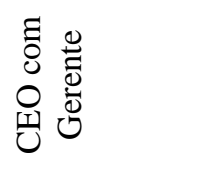 } & Não há Gerente & 114 & 12,3 \\
\hline & & Sim, profissional acumula a atividade clínica e de Gerente do CEO & 351 & 37,7 \\
\hline & & Sim, profissional que atual exclusivamente como Gerente do CEO & 465 & 50,0 \\
\hline
\end{tabular}




\begin{tabular}{lll} 
Total & $\mathbf{9 3 0}$ & $\mathbf{1 0 0}$ \\
Menos de um ano & 249 & 26,8 \\
1 a 2 anos & 315 & 33,9 \\
3 a 4 anos & 87 & 9,4 \\
5 a 9 anos & 111 & 11,9 \\
10 anos ou mais & 8 & 0,9 \\
Não sabe/Não se aplica & 160 & 17,2 \\
Total & $\mathbf{9 3 0}$ & $\mathbf{1 0 0}$ \\
Não & 215 & 23,1 \\
Sim, em gestão pública & 137 & 14,7 \\
Sim, em saúde coletiva & 173 & 18,6 \\
Sim, outros & 291 & 31,3 \\
Não se aplica & 114 & 12,3 \\
Total & $\mathbf{9 3 0}$ & $\mathbf{1 0 0}$ \\
\hline
\end{tabular}

Fonte: Banco de dados do primeiro ciclo PMAQ-CEO, 2014.

\section{Características avaliativas dos municípios}

Três das quatro características analisadas dos municípios foram estatisticamente significantes $(\mathrm{p} \leq$ 0,05) quando comparadas com o relato da realização de alguma atividade de planejamento, nos últimos 12 meses. Os CEO do Centro-Oeste (88,7 \%) tenderam a realizar planejamento das ações. Por outro lado, embora no Nordeste esteja o maior número de CEO (355) este apresenta o segundo mais baixo percentual $(73,2 \%)$ de serviços que realizaram alguma atividade de planejamento. Ademais aqueles CEO que relataram a realização do planejamento estão em municípios com cobertura populacional estimada de saúde bucal na Estratégia Saúde da Família menor ou igual a $63 \%$ e com Índice de Desenvolvimento Humano municipal (IDHM) maior ou igual a 0,7 quando comparados aos que não estão (Tabela 2). 
TABELA 2

REALIZAÇÃO DE ALGUMA ATIVIDADE DE PLANEJAMENTO, NOS ÚLTIMOS 12 MESES, SEGUNDO CARACTERÍSTICAS AVALIATIVAS DO MUNICÍPIO. BRASIL

\begin{tabular}{|c|c|c|c|c|c|c|c|}
\hline \multirow{3}{*}{$\begin{array}{l}\text { Características } \\
\text { Avaliativas do Município }\end{array}$} & \multicolumn{4}{|c|}{$\begin{array}{c}\text { Realização de alguma atividade de } \\
\text { planejamento }\end{array}$} & \multirow{2}{*}{\multicolumn{2}{|c|}{ Total }} & \multirow{3}{*}{$\begin{array}{l}\text { Valor de } \\
\quad \text { p }\end{array}$} \\
\hline & \multicolumn{2}{|c|}{ Não } & \multicolumn{2}{|c|}{ Sim } & & & \\
\hline & $\mathbf{N}$ & $\%$ & $\mathbf{N}$ & $\%$ & $\mathbf{N}$ & $\%$ & \\
\hline Região & & & & & & & $0,005^{* \diamond}$ \\
\hline Centro-Oeste & 7 & 11,3 & 55 & 88,7 & 62 & 100,0 & \\
\hline Nordeste & 95 & 26,8 & 260 & 73,2 & 355 & 100,0 & \\
\hline Norte & 19 & 32,2 & 40 & 67,8 & 59 & 100,0 & \\
\hline Sudeste & 65 & 19,3 & 272 & 80,7 & 337 & 100,0 & \\
\hline Sul & 21 & 17,9 & 96 & 82,1 & 117 & 100,0 & \\
\hline Total & 207 & 22,3 & 723 & $\mathbf{7 7 , 7}$ & 930 & 100,0 & \\
\hline Porte Populacional & & & & & & & $0,410 *$ \\
\hline $0-30.000$ & 60 & 25,8 & 173 & 74,2 & 233 & 100,0 & \\
\hline $30.001-50.000$ & 37 & 23,1 & 123 & 76,9 & 160 & 100,0 & \\
\hline $50.001-100.000$ & 37 & 21,6 & 134 & 78,4 & 171 & 100,0 & \\
\hline$>100.000$ & 73 & 19,9 & 293 & 80,1 & 366 & 100,0 & \\
\hline Total & 207 & 22,3 & 723 & 77,7 & 930 & 100,0 & \\
\hline \multicolumn{7}{|c|}{ Proporção de Cobertura Populacional Estimada SB/eSF } & $0,037 * \diamond$ \\
\hline$\leq 63 \%$ & 111 & 20,1 & 440 & 79,9 & 551 & 59,2 & \\
\hline$>63 \%$ & 96 & 25,3 & 283 & 74,7 & 379 & 40,8 & \\
\hline Total & 207 & 22,3 & 723 & $\mathbf{7 7 , 7}$ & 930 & 100,0 & \\
\hline \multicolumn{7}{|c|}{ Índice de Desenvolvimento Humano municipal (IDHM) } & $0,032 * * \diamond$ \\
\hline$<0,7$ & 96 & 25,5 & 281 & 74,5 & 377 & 100,0 & \\
\hline$\geq 0,7$ & 111 & 20,1 & 442 & 79,9 & 553 & 100,0 & \\
\hline Total & 207 & 22,3 & 723 & 77,7 & 930 & 100,0 & \\
\hline
\end{tabular}

Fonte: Banco de dados do primeiro ciclo PMAQ-CEO, 2014.

* Teste do qui-quadrado de Pearson

** Teste exato de Fisher

${ }^{\diamond}$ Significância estatística alcançada

\section{Características avaliativas dos serviços}

Dentre as características dos serviços analisadas, houve diferenças estatisticamente significante (p $\leq 0,05)$ com a "Realização de autoavaliação" e "Monitoramento e análise das metas". Os serviços que relataram a execução do planejamento tenderam a realizar atividades de autoavaliação $(87,6$ $\%$ ) bem como o monitoramento e análises das metas de cada especialidade (83,9\%) (Tabela 3). 
TABELA 3

REALIZAÇÃO DE ALGUMA ATIVIDADE DE PLANEJAMENTO, NOS ÚlTIMOS 12 MESES SEGUNDO CARACTERÍSTICAS AVALIATIVAS DO SERVIÇO, BRASIL

\begin{tabular}{|c|c|c|c|c|c|c|c|}
\hline \multirow{3}{*}{$\begin{array}{l}\text { Características } \\
\text { Avaliativas do Serviço }\end{array}$} & \multicolumn{4}{|c|}{$\begin{array}{l}\text { Realização de alguma atividade de } \\
\text { planejamento }\end{array}$} & \multirow{2}{*}{\multicolumn{2}{|c|}{ Total }} & \multirow{3}{*}{$\begin{array}{c}\text { Valor de } \\
\quad \text { p }\end{array}$} \\
\hline & \multicolumn{2}{|c|}{ Não } & \multicolumn{2}{|c|}{ Sim } & & & \\
\hline & $\mathbf{N}$ & $\%$ & $\mathbf{N}$ & $\%$ & $\mathbf{N}$ & $\%$ & \\
\hline \multicolumn{7}{|l|}{ Tipo do CEO } & \multirow[t]{5}{*}{$0,133 *$} \\
\hline 1 & 90 & 25,8 & 259 & 74,2 & 349 & 100,0 & \\
\hline 2 & 95 & 20,2 & 375 & 79,8 & 470 & 100,0 & \\
\hline 3 & 22 & 19,8 & 89 & 80,2 & 111 & 100,0 & \\
\hline Total & 207 & 22,3 & 723 & $\mathbf{7 7 , 7}$ & 930 & 100,0 & \\
\hline \multicolumn{7}{|c|}{ Realização de autoavaliação } & \multirow[t]{4}{*}{$0,000 * * \diamond$} \\
\hline Não & 123 & 49,2 & 127 & 50,8 & 250 & 100,0 & \\
\hline Sim & 84 & 12,4 & 596 & 87,6 & 680 & 100,0 & \\
\hline Total & 207 & 22,3 & 723 & 77,7 & 930 & 100,0 & \\
\hline \multicolumn{7}{|c|}{ Monitoramento e análise das metas } & \multirow[t]{4}{*}{$0,000 * \vartheta$} \\
\hline Não & 79 & 58,1 & 57 & 41,9 & 136 & 100,0 & \\
\hline Sim & 128 & 16,1 & 666 & 83,9 & 794 & 100,0 & \\
\hline Total & 207 & 22,3 & 723 & 77,7 & 930 & 100,0 & \\
\hline
\end{tabular}

Fonte: Banco de dados do primeiro ciclo PMAQ-CEO, 2014.

*Teste do qui-quadrado de Pearson

**Teste exato de Fisher

${ }^{\diamond}$ Significância estatística alcançada

\section{Características avaliativas dos gestores dos serviços}

Quanto a análise estatística entre as características dos gestores dos serviços e o relato da realização alguma atividade de planejamento, nos últimos 12 meses, verificou-se diferença significativa ( $\mathrm{p} \leq$ 0,05). A existência de um profissional que atua exclusivamente como gerente do CEO teve associação com a realização do planejamento no serviço quando comparado aos que não tem gerente ou que o mesmo acumula função clínica e de gestão. O tempo de atuação como gerente no CEO e a sua formação complementar na área de gestão pública (83,9 \%) ou em saúde coletiva $(83,8 \%)$ também tiveram associação positiva com a realização de alguma atividade de 
planejamento. Sendo que, os que atuam menos de um ano com o gerente são os que realizam menos atividade de planejamento $(73,1 \%)$ (Tabela 4$)$.

TABELA 4

REALIZAÇÃO DE ALGUMA ATIVIDADE DE PLANEJAMENTO, NOS ÚLTIMOS 12 MESES SEGUNDO CARACTERÍSTICAS AVALIATIVAS DO GESTOR DO SERVIÇO, BRASIL

\begin{tabular}{|c|c|c|c|c|c|c|c|}
\hline \multirow{3}{*}{ Características Avaliativas do Gestor do Serviço } & \multicolumn{4}{|c|}{$\begin{array}{c}\text { Realização de alguma } \\
\text { atividade de planejamento }\end{array}$} & \multirow{2}{*}{\multicolumn{2}{|c|}{ Total }} & \multirow{3}{*}{$\begin{array}{l}\text { Valor } \\
\text { de } p\end{array}$} \\
\hline & \multicolumn{2}{|c|}{ Não } & \multicolumn{2}{|c|}{ Sim } & & & \\
\hline & $\mathbf{N}$. & $\%$ & $\mathbf{N}$. & $\%$ & $\mathbf{N}$. & $\%$ & \\
\hline Existência de gerente no CEO & & & & & & & $0,000 * 0$ \\
\hline Não há gerente & 42 & 36,8 & 72 & 63,2 & 114 & 100,0 & \\
\hline $\begin{array}{l}\text { Sim, profissional acumula a atividade clínica e de } \\
\text { gerente do CEO }\end{array}$ & 74 & 21,1 & 277 & 78,9 & 351 & 100,0 & \\
\hline $\begin{array}{l}\text { Sim, profissional que atual exclusivamente como } \\
\text { gerente do CEO }\end{array}$ & 91 & 19,6 & 374 & 80,4 & 465 & 100,0 & \\
\hline Total & 207 & 22,3 & 723 & $\mathbf{7 7 , 7}$ & 930 & 100,0 & \\
\hline Tempo de atuação no CEO & & & & & & & $0,000 * \diamond$ \\
\hline Não sabe/Não se aplica & 55 & 34,4 & 105 & 65,6 & 160 & 100,0 & \\
\hline Menos de um ano & 67 & 26,9 & 182 & 73,1 & 249 & 100,0 & \\
\hline 1 a 2 anos & 56 & 17,8 & 259 & 82,2 & 315 & 100,0 & \\
\hline 3 a 4 anos & 7 & 8,0 & 80 & 92,0 & 87 & 100,0 & \\
\hline 5 a 9 anos & 21 & 18,9 & 90 & 81,1 & 111 & 100,0 & \\
\hline 10 anos ou mais & 1 & 12,5 & 7 & 87,5 & 8 & 100,0 & \\
\hline Total & 207 & 22,3 & 723 & $\mathbf{7 7 , 7}$ & 930 & 100,0 & \\
\hline Formação complementar & & & & & & & $0,000 * \diamond$ \\
\hline Não se aplica & 42 & 36,8 & 72 & 63,2 & 114 & 100,0 & \\
\hline Não & 58 & 27,0 & 157 & 73,0 & 215 & 100,0 & \\
\hline Sim, em gestão pública & 22 & 16,1 & 115 & 83,9 & 137 & 100,0 & \\
\hline Sim, em saúde coletiva & 28 & 16,2 & 145 & 83,8 & 173 & 100,0 & \\
\hline Sim, outros & 57 & 19,6 & 234 & 80,4 & 291 & 100,0 & \\
\hline Total & 207 & 22,3 & 723 & 77,7 & 930 & 100,0 & \\
\hline
\end{tabular}

Fonte: Banco de dados do primeiro ciclo PMAQ-CEO, 2014.

*Teste do qui-quadrado de Pearson

$\diamond$ Significância estatística alcançada

\section{DISCUSSÃO}


A distribuição dos CEO por Região, tendo quase $75 \%$ dos serviços localizados nas Regiões Nordeste (355) e Sudeste (337), acompanha a disposição da densidade demográfica no Brasil, uma vez que a soma de habitantes de ambas corresponde a $69,9 \%$ da população brasileira.

Quanto ao arranjo por porte populacional, 39,4 \% dos CEO estão localizados em municípios com mais de 100 mil habitantes. Embora municípios deste porte correspondam a $5 \%$ dos municípios no Brasil, neles residem 55 \% da população brasileira. Expondo assim a necessidade de ampliação da oferta de serviços especializados em saúde bucal em municípios de grande porte.

No entanto, apenas a implantação desses serviços não é o suficiente para garantir o acesso ao cuidado especializado. Há necessidade, por exemplo, de organizar uma rede hierarquizada tendo isto como instrumento de planejamento para viabilizar o acesso da população aos serviços de saúde (12).

A inserção da saúde bucal na estratégia Saúde da Família visa superar a prática assistencial, executada por um sujeito individual no limitado espaço clínico-cirúrgico, voltada exclusivamente ao cidadão doente. Procura também trabalhar para a reorganização do acesso às ações de saúde, buscando a atenção integral em saúde bucal aos indivíduos e às famílias. A implementação dos CEO pode constituir estratégia relevante com vista à integralidade da atenção no âmbito odontológico (20). Nesse sentido, o acesso e a utilização dos serviços odontológicos especializados reportam ao entendimento de parte do processo de geração da integralidade como eixo norteador dos sistemas de serviços de saúde, tendo em vista que ofertam tratamentos complementares aos cuidados prestados pela atenção básica (21). 
Pesquisas que analisaram a Política Nacional de Saúde Bucal têm revelado que a integralidade, enquanto articulação entre as ações de promoção, prevenção e tratamento, além da interface entre níveis de atenção, é maior quando há maior cobertura adequada das equipes de saúde bucal na atenção básica (20). Porém, a ausência da realização de planejamento nos CEO, como foi possível constatar neste estudo, na maior parte dos municípios que apresentaram as maiores coberturas de saúde bucal na Estratégia Saúde da Família, pode interferir diretamente de forma negativa na articulação da rede de saúde bucal e consequentemente impedindo um cuidado integral dos usuários.

Em estudo realizado por Silva et al. (2015) (11), verificou-se que, embora os gestores compreendam a importância do planejamento enquanto ação prioritária para organizar os serviços, há impedimentos para a realização dessa prática em virtude de alguns eventos denominados por 'incêndios', os quais demandam respostas ágeis, voltadas para a assistência, de forma que as práticas muitas vezes são encaminhadas pelos gestores sem uma reflexão sobre as mesmas.

A realização de avaliações para subsidiar o planejamento e a tomada de decisão é uma alternativa para buscar soluções que visem superar as barreiras de acesso aos serviços de saúde bucal (17).

O processo de planejamento deve-se iniciar com a identificação de problemas, isto é, pela realização de uma autoavaliação, e a partir desse diagnóstico, definem-se estratégias de intervenção para alcançar as mudanças consideradas necessárias (22). 
A autoavaliação é entendida como ponto de partida da fase de desenvolvimento do PMAQ-CEO, uma vez que os processos orientados para a melhoria da qualidade têm início na identificação e reconhecimento das dimensões positivas e problemáticas do trabalho da gestão e das equipes de atenção à saúde (23).

Os resultados sinalizam que quem realiza atividade de planejamento também realiza autoavaliação e o monitoramento e análise das metas. No entanto, um alto percentual realiza planejamento sem realizar autoavaliação, isto é, não faz autoanálise e identificação de problemas previamente, resultando em uma ação pouco reflexiva.

Dos CEO que informaram a não realização da atividade de autoavaliação (250), 50,8 \% relatam terem realizado alguma atividade de planejamento, nos últimos 12 meses. O que demonstra uma grande fragilidade desse processo uma vez que esse reconhecimento tem como propósito verificar a realidade local, identificando as fragilidades e as potencialidades, conduzindo a planejamentos de intervenção para a melhoria do acesso e da qualidade dos serviços (23), ou seja, se planejamos de forma intuitiva, sem uma autocrítica, ou pouco sistematizada, dificulta a socialização ou a institucionalização dos projetos elaborados e consequentemente a alcance das transformações necessárias (24). Estas considerações ganham eco na afirmação de Bodstein (2002) (25) que destaca a importância do desenvolvimento de uma cultura organizacional no SUS que tenha a avaliação como atividade intrínseca e inseparável do planejamento e da gestão.

A eficácia das organizações de saúde também vai depender das relações que estabelecem pessoas, tecnologia, recursos e administração, para realizar a tarefa organizacional de prestação de serviços 
de saúde. Contudo, entre esses fatores, a administração tem um papel determinante, uma vez que ela realiza o trabalho de combinar pessoas, tecnologia e recursos para atingir os objetivos organizacionais, mediante planejamento, coordenação, direção e controle. Essa tarefa de administrar, também identificada como gerenciar, é que possibilitará a organização de saúde, mudar os níveis de atenção, a qualidade de seus serviços (26).

As organizações de saúde necessitam de gerentes que tenham competência para enfrentar os desafios gerados pela complexidade do Setor Saúde e as exigências por qualidade nos serviços prestados à população. Para desempenhar esta função é necessário conhecer o planejamento, as estratégias para intervenção, a programação; entender sobre as formas de contrato, sobre gestão dos processos de trabalho e do conhecimento, entender a organização da rede de saúde, além das questões relacionadas à infraestrutura predial das unidades de saúde e sua manutenção (24).

Na realidade brasileira, principalmente em organizações públicas de saúde, os desafios para uma melhor formação de gerentes são enormes, assemelhando-se aos desafios da própria consolidação do SUS e da estratégia de expansão das Unidades Primárias de Saúde (24).

O planejamento é um instrumento de gestão, no entanto a consolidação da cultura do seu uso nos serviços de saúde, apesar dos avanços atingidos ainda assim é um grande desafio no SUS (27).

\section{CONCLUSÕES}


Os resultados apresentados descrevem e analisam aspectos dos municípios, dos serviços e dos gerentes dos serviços em relação ao planejamento das ações nos CEO relatados durante a Avaliação Externa. Entretanto, a leitura das informações precisa considerar, nesse caso, tanto o limite explicativo dos dados exclusivamente quantitativos, quanto a natureza da coleta que tem desdobramentos políticos e financeiros para a gestão municipal/estadual nos resultados da Avaliação Externa.

Os resultados desta pesquisa demonstraram uma diversidade e uma incompletude na implantação de ferramentas de planejamento pelos CEO do Brasil.

Para a maioria das associações analisadas houve diferença significante estatisticamente. Os CEO que mais relataram a realização de alguma atividade de planejamento estão em sua maioria da Região Centro-Oeste e Sul, estão em municípios com menor cobertura de saúde bucal da estratégia Saúde da Família e em municípios com alto Índice de Desenvolvimento Humano.

Os resultados apontaram também que a existência de gerente no CEO, o seu tempo de atuação no serviço maior do que 2 anos e os que tem uma formação complementar na área de gestão pública ou de saúde coletiva tende a realizar mais atividades de planejamento.

Os resultados demonstram que os CEO que tem gerentes, de preferência com atuação exclusiva na gestão, e que haja investimentos que visem a qualificação da formação destes profissionais. No entanto, o planejamento e autoavaliação não podem ser tratados como ferramentas meramente 
burocráticas e sem sentido, que não envolvam gestores e profissionais diretamente relacionados com o serviço e cuidado prestado.

\section{RECOMENDAÇÕES}

Faz-se necessário que novos estudos acerca do planejamento sejam realizados, tendo em vista sua relevância, no intuito de aprofundar o tema, e, sobretudo para investigar a qualidade desta ação realizada pelos serviços.

\section{REFERÊNCIAS}

1. Noronha JC, Lima LD, Machado CV. A gestão do Sistema Único de Saúde: características e tendências. In: Saúde no Brasil: Contribuições para a Agenda de Prioridades de Pesquisa. Brasília, Brasil: Ministério da Saúde; 2004. P. 306.

2. Vieira FS. Avanços e desafios do planejamento no Sistema Único de Saúde. Ciênc. Saúde Coletiva. 2009: 14(Supl. 1): 1565-77.

3. Ontario Ministry of Health and Long-Term Care. The health planner's toolkit. Ottawa, ON, Canada: Ministry of Health; 2006. P. 38.

4. Buarque SC. Metodologia de planejamento do desenvolvimento local e municipal sustentável. Brasília, Brasil: Instituto Nacional de Colonização e Reforma Agrária; 1999. P. 97.

5. Lawrence D. Strategic approaches to planning health services. In: Pencheon D, Guest C, Melzer D, Muir Gray J (Eds), Oxford handbook of public health practice. Oxford, UK: Oxford University Press; 2006. 
6. RAND Europe. Framework for assessing, improving and enhancing health service planning. Santa Monica, CA, USA: RAND Corporation; 2010. P. 70.

7. Matus C. Política planejamento \& governo. Brasília, Brasil: Instituto de Pesquisa Econômica Aplicada; 1993.

8. Queensland Health. Guide to health service planning (version 3) Brisbane, Australia: Queensland Government; 2015.

9. Green A. An introduction to health planning for developing health systems. Oxford, UK: Oxford University Press; 2007.

10. World Health Organization (WHO), Regional Office for Africa. Planning and implementation of district health services. Brazzaville, Congo: WHO Regional Office for Africa; 2004.

11. Silva BFS, Wandekoken KD, Dalbello-Araujo M, Benito GAV. A importância do planejamento como prática de gestão na microrregião de saúde de São Mateus (ES). Saúde Debate. 2015; 39(104): 183-96.

12. Jesus WLA, Assis MMA. Revisão sistemática sobre o conceito de acesso nos serviços de saúde: contribuições do planejamento. Ciênc Saúde Coletiva. 2010; 15(1): 161-70.

13. Queensland Health. Department of Health integrated planning framework. Brisbane, Australia: Queensland Government; 2013.

14. República Federativa do Brasil, Ministério da Saúde. Programa Nacional de Melhoria do Acesso e da Qualidade da Atenção Básica. Manual Instrutivo: Saúde Mais Perto de Você Acesso e Qualidade. [acesso 23 junho. 2016]. Brasília, Brasil: Ministério da Saúde; 2013. Disponível em: http://189.28.128.100/dab/docs/portaldab/publicacoes/manual_instrutivo_PMAQ_CEO.pdf. 
15. Pisco LA. Avaliação como instrumento de mudança. Ciên Saúde Coletiva. 2006; 11(3): 56668.

16. República Federativa do Brasil, Ministério da Saúde, . Secretaria de Atenção. CoordenaçãoGeral de Saúde Bucal. Portaria GM n. ${ }^{\circ}$ 599/2006. Brasília, Brasil: Ministério da Saúde; 2006. p. 51.

17. Machado FCA, Silva JV, Ferreira MAF. Fatores relacionados ao desempenho de Centros de Especialidades Odontológicas. Ciênc Saúde Coletiva. 2015; 20(4): 1149-163.

18. Kawata LS, Mishima SM, Chirelli MQ, Pereira MJB. O trabalho cotidiano da enfermeira na saúde da família: utilização de ferramentas da gestão. Texto \& Contexto Enferm. 2009; 18(2): $313-20$.

19. Sarti TD, Campos CEA, Zandonade E, Ruschi GEC, Maciel ELN. Avaliação das ações de planejamento em saúde empreendidas por equipes de saúde da família. Cad Saúde Pública. 2012; 28(3): 537-48.

20. Chaves SCL, Barros SG, Cruz DN, Figueiredo ACL, Moura BLA, Cangussu MCT . Política Nacional de Saúde Bucal: fatores associados à integralidade do cuidado. Rev Saúde Pública. 2010; 44(6): 1005-13.

21. Chaves SCL, Soares FF, Rossi TRA, Cangussu MCT, Figueiredo ACL, Cruz DN, Cury PR . Características do acesso e utilização de serviços odontológicos em municípios de médio porte. Ciênc Saúde Coletiva. 2012; 17(11): 3115-24.

22. Chorny AH, Kuschnir R, Taveira M. Planejamento e programação em saúde. [acesso 10 ago. 2016]. Rio de Janeiro, Brasil; Ministério da Saúde, Fundação Oswaldo Cruz, Escola Nacional da Saúde Pública Sergio Arouca; 2008. Disponível em: http://www.ensp.fiocruz.br/portalensp/_uploads/documentos-pessoais/documento-pessoal_12465.pdf. 
23. República Federativa do Brasil, Ministério da Saúde, Secretaria de Atenção à Saúde, Departamento de Atenção Básica. Autoavaliação para a Melhoria do Acesso e da Qualidade dos Centros de Especialidades Odontológicas: AMAQ-CEO. [acesso 23 jun. 2016]. Brasília, Brasil: Ministério da Saúde; 2013. Disponível em: http://189.28.128.100/dab/docs/portaldab/publicacoes/AMAQ_CEO.pdf.

24. Franco CA, Santos SA, Salgado MF. In: Pessôa LR, Santos EHA, Torres KRBO, organizadores. Manual do Gerente: desafios da média gerência na saúde. Rio de Janeiro, Brasil: Escola Nacional da Saúde Pública Sergio Arouca; 2011.

25. Bodstein R. Atenção Básica na agenda da saúde. Ciênc Saúde Coletiva. 2002; 7(3): 401-12.

26. Junqueira LAP. Gerência dos serviços de saúde. Cad Saúde Pública. 1990; 6(3): 247-59.

27. República Federativa do Brasil, Ministério da Saúde, Organização Pan-Americana da Saúde. Sistema de Planejamento do SUS (PlanejaSUS): uma construção coletiva - trajetória e orientações de operacionalização. Brasília, Brasil: Ministério da Saúde; 2009. p. 318. 